\title{
La desigualdad salarial expresada en la dispersión económica del derecho laboral al aguinaldo en México
}

Wage inequality in Mexico expressed in economic dispersion of mandatory year-end bonus

A desigualdade salarial expressa na dispersão econômica do direito ao décimo terceiro salário no México

José Manuel Osorio Atondo

jose.osorio@ues.mx

Universidad Estatal de Sonora - México

https://orcid.org/0000-0001-8508-9953

\section{Danitza María Gastélum Celaya}

danitza.gastelum@ues.mx

Universidad Estatal de Sonora - México

https://orcid.org/0000-0003-1477-4181

\begin{abstract}
RESUMEN
En las economías nacionales la distribución del ingreso ha sido uno de los fenómenos más estudiados desde su contexto económico, político y social; en donde la desigualdad salarial se ha exhibido como uno de los principales motivos que inciden negativamente en el desarrollo y crecimiento en los países. Precisamente, en el presente análisis se ostenta la desigualdad salarial manifestada en la dispersión económica del derecho laboral de los trabajadores al aguinaldo en México, tomando como fundamento la legislación aplicable y los datos disponibles de la Encuesta Nacional de Ingresos y Gastos en los Hogares (ENIGH) por los años 2015, 2016 y 2018; apoyándose metodológicamente para el tratamiento estadístico, descripción y examen de datos en la herramienta de Excel y el solucionador matemático Symbolab, así como mediante la aplicación de la regla de Sturges, la curva de Lorenz y el índice de Gini que muestran finalmente la falta de equidad en tal percepción económica en los hogares de México por los períodos expuestos.
\end{abstract}

Palabras clave: Desigualdad salarial, salario, aguinaldo, derecho laboral.

\begin{abstract}
Income distribution within the economic, political, and social context of the national economies of countries has been examined in numerous and varied studies, showing wage inequality is one of the main factors negatively impacting development and growth. This analysis shows the wage inequality evident in the economic dispersion of constitutionally mandated year-end bonuses (aguinaldo) to workers based on available data gathered in the National Household Income and Spending Survey (ENIGH, by its Spanish-language acronym) for the years 2015, 2016 and 2018. Employing a statistical processing methodology involving, description, and examination of data with the aid of Excel, specifically the Symbolab Math Solver, the study employs the Sturges rule, the Lorenz Curve and the Gini Index, which reveal inequality of the year-end bonus payment to Mexico's households in the years under study.
\end{abstract}

Keywords: Wage inequality, salary, year-end bonus, labor law.

\section{RESUMO}

Nas economias nacionais a distribuição da renda tem sido um dos fenômenos mais estudados a partir do seu contexto econômico, político e social; onde a desigualdade salarial tem demonstrado ser uma das principais razões que afetam negativamente o desenvolvimento e o crescimento nos países. Precisamente, a presente análise mostra a desigualdade salarial manifestada na dispersão econômica do direito trabalhista ao décimo terceiro salário no México, com base na legislação aplicável e nos dados disponíveis da Pesquisa Nacional de Renda e Despesas por Domicílio (ENIGH, na sigla em espanhol) dos anos 2015, 2016 e 2018; contando com o apoio metodológico para o tratamento estatístico, descrição, exame de dados na ferramenta Excel e o solucionador matemático symbolab, bem como através da aplicação da regra de Sturges, da curva de Lorenz e do índice de Gini que mostram finalmente a falta de equidade na percepção econômica nos lares mexicanos nos períodos mencionados.

Palavras chave: Desigualdade salarial, salário, décimo terceiro, direito trabalhista. 


\section{INTRODUCCIÓN}

Paradójicamente en la actualidad el mundo es más rico, sin embargo, más disímil que nunca en los ámbitos de la alimentación, la salud, las oportunidades de empleo, la distribución del ingreso, entre otras manifestaciones que inciden sin lugar a duda en la pobreza y muchos de los países en situación extrema.

En el contexto económico, la concentración de la riqueza o bien la desigualdad de los ingresos está en aumento, en virtud de que el 10\% más rico la población mundial obtiene hasta el $40 \%$ del ingreso total (ONU), escenario que obedece a empleos informales, desempleo, bajos salarios, carencia de educación, alza de precios y políticas públicas que no coadyuvan en equilibrar las precepciones económicas en los hogares.

Por tal motivo, en el presente estudio se exhibe uno de los elementos de la desigualdad salarial manifestada en la dispersión económica del derecho laboral al aguinaldo en México; exponiendo en primer término la descripción del fenómeno de estudio, la identificación en el marco jurídico del derecho a dicha remuneración en las relaciones individuales o colectivas de trabajo, el tratamiento estadístico de datos emitidos en la Encuesta Nacional de Ingresos y Gastos en los Hogares (ENIGH) por los años 2015, 2016 y 2018 y los resultados obtenidos en la curva de Lorenz y determinación del índice de Gini.

\section{FUNDAMENTACIÓN TEÓRICA}

\subsection{La desigualdad salarial}

El tema de la desigualdad como tópico central y en sus distintos escenarios o factores que inciden en la misma, ha sido abordado en varios países; tales como la composición de los hogares, el acceso a la educación, a la seguridad social, a la salud, el ingreso, la pobreza, el empleo, la brecha salarial por género, entre otros elementos que coadyuvan en el análisis de la inequidad que se enfrenta en cualquier sociedad.

De tal manera que la desigualdad ha simbolizado uno de las temáticas mayormente controversiales desde el contexto económico, político y social, primordialmente en sus principales dimensiones: la distribución de la riqueza y del ingreso (Navarrete, 2016:45), simbolizando un fenómeno complejo y considerada como un elemento natural con pocas probabilidades de erradicarse cabalmente, la cual ha prevalecido desde hace siglos e intensificándose con la llegada de modelos económicos como el capitalismo que estimula el acaparamiento de riquezas, no obstante, se deben de indagar mecanismos para mitigar sus efectos (Carrera, Villafuerte y Rodríguez, 2017: 15 y 16).

En esta misma tesitura y específicamente en la distribución del ingreso, el Banco Mundial (BM), comparte que el nivel de desigualdad de una nación, representa una dimensión trascendental del bienestar, al considerar las diferencias en el ingreso domésticos de toda la población e indicador que muestra elementos para la implementación de políticas gubernamentales orientadas a la reducción de la pobreza. 
Para el caso que nos atañe, en México se han efectuado una serie de análisis sobre el comportamiento del empleo, la distribución del ingreso y/o estructura salarial, partiendo primordialmente en el inicio de cambios estructurales en el país; estudios que se han apoyado en diferentes bases de datos e instrumentos disponibles y emitidos por dependencias gubernamentales u organismos nacionales, en donde destacan la Encuesta Nacional de Empleo Urbano (ENEU), Encuesta Nacional de Ingreso Gasto de los Hogares (ENIGH), Encuesta Nacional de Empleo (ENE), Censos Económicos (CE), Encuesta Industrial Mensual (EIM), Encuesta Nacional de Empleo, Salario, Tecnología y Capacitación (ENESTYC), entre otros (Castro y Huesca, 2007: 234).

Verbigracia, existen estudios empíricos que exhiben la brecha o desigualdad salarial con enfoque de género en correlación a los contextos de la discriminación y el capital humano (Jasso y Acevedo, 2004) así como sus incidencias en los ámbitos culturares y sociales (Araújo, 2015). Asimismo, análisis que exteriorizan desde la misma perspectiva de género, pero en contextos regionales que muestran la amplia brecha inequitativa de la distribución del ingreso en México (Llamas, Huesca y Gutiérrez, 2019; Rodríguez, 2018; Rodríguez y Limas, 2017).

En esta misma postura y en atención a los análisis del BM mediante su instrumento "LAC Equity Lab", en los años 2016 y 2018 el centil 100 tenía el $10.3 \%$ y $11.0 \%$ del ingreso total respectivamente y que correspondía en la categoría de los ricos en México. Asimismo, es considerado como el país con el nivel más alto de desigualdad del ingreso en su población, escenario en el cual el 20\% más rico gana diez veces más que los que se hallan en la parte inferior de la distribución del ingreso; así con el menor ingreso disponible promedio de los hogares según el reporte de la Organización para la Cooperación y Desarrollo Económico (OCDE) (Hernández, 2020).

\subsection{El aguinaldo}

En atención a la Declaración Universal de Derechos Humanos (DUDH) emitida por la Organización de las Nacionales Unidas (ONU), instituye en su artículo 23 que "toda persona que trabaja tiene derecho a una remuneración equitativa y satisfactoria que le asegure, así como a su familia, una existencia conforme a la dignidad humana", por lo que tal percepción económica debe permitir cubrir las necesidades básicas de alimentación, salud, vivienda, educación en lo individual como en lo familiar, constituyéndose el salario como uno de los derechos fundamentales (CNDH) y estipulándose en las legislaciones nacionales una percepción mínima diaria.

Precisamente en correspondencia a la Constitución Política de los Estados Unidos Mexicanos (CPEUM), se fundamente en su numeral 123 fracción IV, que el salario mínimo deberá ser "suficiente para satisfacer las necesidades normales de un jefe de familia, en el orden material, social y cultural, y para proveer a la educación obligatoria de los hijos".

De acuerdo a la Ley Federal del Trabajo (LFT), el salario se define como la remuneración que retribuye el patrón al trabajador por su trabajo, mismo que se integra por los pagos de cuota diaria, gratificaciones, percepciones, habitación, primas, comisiones, prestaciones en especia y cualquier otra cantidad o prestación que se entregue al trabajador por su labor. Es preciso señalar, que, aunque se estipula un mínimo, el trabajador puede obtener una remuneración superior en atención a las funciones que desempeña; sin embargo, existen las prestaciones mínimas con base a dicha norma jurídica, en las que destacan el disfrute de vacaciones pagadas, la prima vacacional y el aguinaldo. 
Según la Real Academia Española (REA), define al aguinaldo como "Regalo que se da en Navidad o en la fiesta de la Epifanía”. Al respecto, Sánchez (2009:716 y 717) comparte que el aguinaldo o paga extraordinaria es una práctica muy longeva que tiene su génesis en el pueblo celta, costumbre identificada como "eguinad", donde la comunidad designaba el regalo de año nuevo. Subsiguientemente se institucionalizó en Roma, surgiendo a la postre el concepto de la paga extraordinaria en dinero o en especie cuando el patrón deseaba premiar al trabajador según las diferentes culturas.

Justamente en el artículo 87 de la LFT, a la letra señala: "Los trabajadores tendrán derecho a un aguinaldo anual que deberá pagarse antes del día veinte de diciembre, equivalente a quince días de salario, por lo menos". Es decir, tal gratificación se determina en función del salario diario que percibe el trabajador y de los días que determine la relación laboral individual o colectiva sin ser inferior a quince días. Es preciso señalar, que, en las relaciones laborales del sector público en México, perciben en algunos casos hasta 60 días de tal percepción económica.

\section{PROCEDIMENTOS METODOLÓGICOS}

Para el desarrollo de la investigación se basó en una metodología mixta exploratoria mediante el análisis de tipo documental, descriptivo, deductivo y cuantitativo; a través del examen de documentos de carácter jurídico, legislación aplicable y legajos técnicos en el tema de estudio e inquisiciones disponibles sobre la materia. Se utilizaron métodos de localización, fijación y tratamiento de datos y cifras emitidas por el Instituto Nacional de Estadística y Geografía (INEGI) con sus resultados exteriorizados en el instrumento denominado Encuesta Nacional de Ingresos y Gastos en los Hogares (ENIGH), específicamente en la variable analizada e identificada como P009 y que refiere al aguinaldo por los años 2015, 2016 y 2018 por familia, lo que indica que puede haber más de una persona por hogar que recibió dicha percepción económica.

De los años 2015 y 2016 se analizaron 33059 y 37956 datos respectivamente agrupados en 17 intervalos por año. Para el 2018, se analizaron 13,838 datos, agrupados en 15 clases o intervalos, los cuales se determinaron con base en la regla de Sturges (1926).

$\mathrm{I}=1+\frac{\log (\mathrm{M})}{\log (2)}$, donde $\mathrm{M}$ es el tamaño de la muestra

Tabla 1. Determinación de clases conforme a la regla de Sturges.

\begin{tabular}{|c|c|c|c|}
\hline Año & Hogares ENIGH & Regla de Sturges & *Redondeo \\
\hline 2015 & 33059 & $I=1+\frac{\log (33059)}{\log (2)}=16.0130804$ & 17 \\
\hline 2016 & 37956 & $I=1+\frac{\log (37956)}{\log (2)}=16.21236961$ & 17 \\
\hline 2018 & 13,838 & $I=1+\frac{\log (13838)}{\log (2)}=14.7566456$ & 15 \\
\hline
\end{tabular}

Fuente: Elaboración propia con datos emitidos en la ENIGH 2015, 2016 y 2018 y la regla de Sturges.

* Debido a que el entero del resultado previo al redondeo es par, por ende, se aumenta al alta. 
El valor máximo y mínimo de aguinaldo permiten determinar el rango $(\mathrm{R})$ del conjunto de datos, este cálculo resulta de la diferencia entre el dato mayor y el dato menor. $R=\operatorname{Max}-\operatorname{Min}$.

Posteriormente se determina la amplitud de cada intervalo (A), el cual se obtiene del cociente del rango y el número de intervalos: $A=\frac{R}{I}$

Seguidamente, se calculó la marca de clase (X), que es el promedio que hay entre los dos límites superior e inferior, continuando con el cálculo de la frecuencia absoluta (fi). La frecuencia absoluta es el número de veces que aparece un determinado dato estadístico. Con el fin de facilitar este cálculo, se utilizó la Función Frecuencia en Excel, la cual requiere los parámetros datos y grupos: Frecuencia (datos, grupos). En el presente estudio el parámetro datos serían el total de familias como datos analizados; el segundo parámetro corresponde a los grupos o clases con los límites superiores e inferiores ya calculados. Al estimar la frecuencia absoluta de todos los intervalos y sumar dichas frecuencias, esta debe dar el total de datos (M).

$\{=$ FRECUENCIA (datos de la muestra, grupos de intervalos) $\}$

Subsiguientemente se calculó la frecuencia absoluta acumulada (Fi) que es la suma de la frecuencia del dato más la frecuencia del o los datos anteriores, permitiendo con ello estimar las frecuencias relativas (hi), la cual es la división de la frecuencia absoluta entre el total de datos (M).

$h i=\frac{f i}{M}$

Una vez calculada la frecuencia relativa, se sumaron los resultados, arrojando un total de 1. Postreramente se determinó la frecuencia relativa acumulada (hi acumulada), el acumulado por nivel, el \% por nivel y el \% acumulado por nivel: La hi acumulado resultó de la suma de la frecuencia relativa del intervalo más la frecuencia relativa del intervalo anterior. El acumulado por nivel es el producto del promedio de cada intervalo (marca de clase) por la frecuencia absoluta (fi). De igual forma el \% acumulado por nivel se estimó dividiendo el acumulado de cada intervalo entre el acumulado por nivel. Finalmente, se calculó el \% acumulado por nivel, obteniéndose las siguientes tablas que muestran los resultados en el procesamiento de los datos en cada uno de los períodos analizados.

Tabla 2. Máximos, mínimos y amplitud de cada intervalo.

\begin{tabular}{|c|c|c|c|}
\hline Elemento & $\mathbf{2 0 1 5}$ & $\mathbf{2 0 1 6}$ & $\mathbf{2 0 1 8}$ \\
\hline Muestra (M) & 33059 & 37956 & 13838 \\
\hline Max & $\$ 500,000.00$ & $\$ 162,139.00$ & $\$ 487,500.00$ \\
\hline Min & $\$ 1.00$ & $\$ 4.00$ & $\$ 5030.00$ \\
\hline Número de intervalos (I) & 16.0130804 & 16.2123696 & 14.7566456 \\
\hline Amplitud (A) & 29412 & 9537 & 32165 \\
\hline
\end{tabular}

Fuente: Elaboración propia con datos emitidos en la ENIGH, 2015, 2016 y 2018.

Tabla 3. Marca de clase y frecuencias por intervalo 2015.

\begin{tabular}{|r|r|r|r|r|r|r|r|r|r|r|}
\hline $\mathbf{I}$ & $\begin{array}{c}\text { Límite } \\
\text { inferior }\end{array}$ & \multicolumn{1}{c|}{$\begin{array}{c}\text { Límite } \\
\text { superior }\end{array}$} & $\begin{array}{c}\text { X } \\
\text { (marca de } \\
\text { clase) }\end{array}$ & \multicolumn{1}{c|}{$\mathbf{f i}$} & \multicolumn{1}{|c|}{$\mathbf{F i}$} & \multicolumn{1}{c|}{ hi } & $\begin{array}{c}\text { hi } \\
\text { acumulada }\end{array}$ & $\begin{array}{c}\text { Acumulado } \\
\text { por nivel }\end{array}$ & $\begin{array}{c}\text { \% por } \\
\text { nivel }\end{array}$ & $\begin{array}{c}\text { \% } \\
\text { acumulado } \\
\text { por nivel }\end{array}$ \\
\hline $\mathbf{0}$ & 0 & 0 & 0 & 0 & 0 & 0 & 0 & 0 & 0 & 0 \\
\hline $\mathbf{1}$ & 1 & 29413 & $\$ 14,706.85$ & 31955 & 31955 & $96.66 \%$ & $96.7 \%$ & $\$ 469,957,486$ & $89.5 \%$ & $89.5 \%$ \\
\hline $\mathbf{2}$ & 29414 & 58825 & $\$ 44,119.56$ & 949 & 32904 & $2.87 \%$ & $99.5 \%$ & $\$ 41,869,461$ & $8.0 \%$ & $97.5 \%$ \\
\hline $\mathbf{3}$ & 58826 & 88238 & $\$ 73,532.26$ & 126 & 33030 & $0.38 \%$ & $99.9 \%$ & $\$ 9,265,065$ & $1.8 \%$ & $99.3 \%$ \\
\hline $\mathbf{4}$ & 88239 & 117651 & $\$ 102,944.97$ & 15 & 33045 & $0.05 \%$ & $100.0 \%$ & $\$ 1,544,175$ & $0.3 \%$ & $99.6 \%$ \\
\hline
\end{tabular}




\begin{tabular}{|r|r|r|r|r|r|r|r|r|r|r|}
\hline $\mathbf{5}$ & 117652 & 147064 & $\$ 132,357.68$ & 10 & 33055 & $0.03 \%$ & $100.0 \%$ & $\$ 1,323,577$ & $0.3 \%$ & $99.8 \%$ \\
\hline $\mathbf{6}$ & 147065 & 176476 & $\$ 161,770.38$ & 2 & 33057 & $0.01 \%$ & $100.0 \%$ & $\$ 323,541$ & $0.1 \%$ & $99.9 \%$ \\
\hline $\mathbf{7}$ & 176477 & 205889 & $\$ 191,183.09$ & 1 & 33058 & $0.00 \%$ & $100.0 \%$ & $\$ 191,183$ & $0.0 \%$ & $99.9 \%$ \\
\hline $\mathbf{8}$ & 205890 & 235302 & $\$ 220,595.79$ & 0 & 33058 & $0.00 \%$ & $100.0 \%$ & $\$ 0$ & $0.0 \%$ & $99.9 \%$ \\
\hline $\mathbf{9}$ & 235303 & 264714 & $\$ 250,008.50$ & 0 & 33058 & $0.00 \%$ & $100.0 \%$ & $\$ 0$ & $0.0 \%$ & $99.9 \%$ \\
\hline $\mathbf{1 0}$ & 264715 & 294127 & $\$ 279,421.21$ & 0 & 33058 & $0.00 \%$ & $100.0 \%$ & $\$ 0$ & $0.0 \%$ & $99.9 \%$ \\
\hline $\mathbf{1 1}$ & 294128 & 323540 & $\$ 308,833.91$ & 0 & 33058 & $0.00 \%$ & $100.0 \%$ & $\$ 0$ & $0.0 \%$ & $99.9 \%$ \\
\hline $\mathbf{1 2}$ & 323541 & 352952 & $\$ 338,246.62$ & 0 & 33058 & $0.00 \%$ & $100.0 \%$ & $\$ 0$ & $0.0 \%$ & $99.9 \%$ \\
\hline $\mathbf{1 3}$ & 352953 & 382365 & $\$ 367,659.32$ & 0 & 33058 & $0.00 \%$ & $100.0 \%$ & $\$ 0$ & $0.0 \%$ & $99.9 \%$ \\
\hline $\mathbf{1 4}$ & 382366 & 411778 & $\$ 397,072.03$ & 0 & 33058 & $0.00 \%$ & $100.0 \%$ & $\$ 0$ & $0.0 \%$ & $99.9 \%$ \\
\hline $\mathbf{1 5}$ & 411779 & 441191 & $\$ 426,484.74$ & 0 & 33058 & $0.00 \%$ & $100.0 \%$ & $\$ 0$ & $0.0 \%$ & $99.9 \%$ \\
\hline $\mathbf{1 6}$ & 441192 & 470603 & $\$ 455,897.44$ & 0 & 33058 & $0.00 \%$ & $100.0 \%$ & $\$ 0$ & $0.0 \%$ & $99.9 \%$ \\
\hline $\mathbf{1 7}$ & 470604 & 500016 & $\$ 485,310.15$ & 1 & 33059 & $0.00 \%$ & $100.0 \%$ & $\$ 485,310$ & $0.1 \%$ & $100.0 \%$ \\
\hline & & & & $\mathbf{3 3 0 5 9}$ & & $100.00 \%$ & & & $100.0 \%$ & \\
\hline
\end{tabular}

Fuente: Elaboración propia con datos emitidos en la ENIGH 2015.

Tabla 5. Marca de clase y frecuencias por intervalo 2016.

\begin{tabular}{|r|l|r|r|r|r|r|r|r|r|r|}
\hline $\mathbf{I}$ & $\begin{array}{c}\text { Límite } \\
\text { inferior }\end{array}$ & $\begin{array}{c}\text { Límite } \\
\text { superior }\end{array}$ & $\begin{array}{c}\text { X } \\
\text { (marca de } \\
\text { clase) }\end{array}$ & \multicolumn{1}{|c|}{$\mathbf{f i}$} & \multicolumn{1}{|c|}{$\mathbf{F i}$} & \multicolumn{1}{c|}{$\mathbf{h i}$} & $\begin{array}{c}\text { hi } \\
\text { acumulada }\end{array}$ & $\begin{array}{c}\text { Acumulado } \\
\text { por nivel }\end{array}$ & $\begin{array}{c}\text { \% por } \\
\text { nivel }\end{array}$ & $\begin{array}{c}\text { \% } \\
\text { acumulado } \\
\text { por nivel }\end{array}$ \\
\hline $\mathbf{0}$ & 0 & 0 & 0 & & 0 & 0 & 0 & 0 & 0 & 0 \\
\hline $\mathbf{1}$ & 4 & 9541 & $\$ 4,772.67$ & 37597 & 37597 & $99.05 \%$ & $99.1 \%$ & $\$ 179,437,933$ & $96.8 \%$ & $96.8 \%$ \\
\hline $\mathbf{2}$ & 9542 & 19080 & $\$ 14,311.00$ & 316 & 37913 & $0.83 \%$ & $99.9 \%$ & $\$ 4,522,276$ & $2.4 \%$ & $99.3 \%$ \\
\hline $\mathbf{3}$ & 19081 & 28618 & $\$ 23,849.33$ & 32 & 37945 & $0.08 \%$ & $100.0 \%$ & $\$ 763,179$ & $0.4 \%$ & $99.7 \%$ \\
\hline $\mathbf{4}$ & 28619 & 38156 & $\$ 33,387.66$ & 4 & 37949 & $0.01 \%$ & $100.0 \%$ & $\$ 133,551$ & $0.1 \%$ & $99.8 \%$ \\
\hline $\mathbf{5}$ & 38157 & 47695 & $\$ 42,926.00$ & 4 & 37953 & $0.01 \%$ & $100.0 \%$ & $\$ 171,704$ & $0.1 \%$ & $99.8 \%$ \\
\hline $\mathbf{6}$ & 47696 & 57233 & $\$ 52,464.33$ & 0 & 37953 & $0.00 \%$ & $100.0 \%$ & $\$ 0$ & $0.0 \%$ & $99.8 \%$ \\
\hline $\mathbf{7}$ & 57234 & 66771 & $\$ 62,002.66$ & 2 & 37955 & $0.01 \%$ & $100.0 \%$ & $\$ 124,005$ & $0.1 \%$ & $99.9 \%$ \\
\hline $\mathbf{8}$ & 66772 & 76310 & $\$ 71,540.99$ & 0 & 37955 & $0.00 \%$ & $100.0 \%$ & $\$ 0$ & $0.0 \%$ & $99.9 \%$ \\
\hline $\mathbf{9}$ & 76311 & 85848 & $\$ 81,079.33$ & 0 & 37955 & $0.00 \%$ & $100.0 \%$ & $\$ 0$ & $0.0 \%$ & $99.9 \%$ \\
\hline $\mathbf{1 0}$ & 85849 & 95386 & $\$ 90,617.66$ & 0 & 37955 & $0.00 \%$ & $100.0 \%$ & $\$ 0$ & $0.0 \%$ & $99.9 \%$ \\
\hline $\mathbf{1 1}$ & 95387 & 104925 & $\$ 100,155.99$ & 0 & 37955 & $0.00 \%$ & $100.0 \%$ & $\$ 0$ & $0.0 \%$ & $99.9 \%$ \\
\hline $\mathbf{1 2}$ & 104926 & 114463 & $\$ 109,694.32$ & 0 & 37955 & $0.00 \%$ & $100.0 \%$ & $\$ 0$ & $0.0 \%$ & $99.9 \%$ \\
\hline $\mathbf{1 3}$ & 114464 & 124001 & $\$ 119,232.66$ & 0 & 37955 & $0.00 \%$ & $100.0 \%$ & $\$ 0$ & $0.0 \%$ & $99.9 \%$ \\
\hline $\mathbf{1 4}$ & 124002 & 133540 & $\$ 128,770.99$ & 0 & 37955 & $0.00 \%$ & $100.0 \%$ & $\$ 0$ & $0.0 \%$ & $99.9 \%$ \\
\hline $\mathbf{1 5}$ & 133541 & 143078 & $\$ 138,309.32$ & 0 & 37955 & $0.00 \%$ & $100.0 \%$ & $\$ 0$ & $0.0 \%$ & $99.9 \%$ \\
\hline $\mathbf{1 6}$ & 143079 & 152616 & $\$ 147,847.65$ & 0 & 37955 & $0.00 \%$ & $100.0 \%$ & $\$ 0$ & $0.0 \%$ & $99.9 \%$ \\
\hline $\mathbf{1 7}$ & 152617 & 162155 & $\$ 157,385.99$ & 1 & 37956 & $0.00 \%$ & $100.0 \%$ & $\$ 157,386$ & $0.1 \%$ & $100.0 \%$ \\
\hline & & & & $\mathbf{3 7 9 5 6}$ & & $100.00 \%$ & & & $100.0 \%$ & \\
\hline
\end{tabular}

Fuente: Elaboración propia con datos emitidos en la ENIGH 2016.

Tabla 6. Marca de clase y frecuencias por intervalo 2018.

\begin{tabular}{|c|c|c|c|c|c|c|c|c|c|c|}
\hline $\mathbf{I}$ & $\begin{array}{l}\text { Límite } \\
\text { inferior }\end{array}$ & $\begin{array}{c}\text { Límite } \\
\text { superior }\end{array}$ & $\begin{array}{c}\mathrm{X} \\
\text { (marca de } \\
\text { clase) }\end{array}$ & fi & $\mathbf{F i}$ & hi & $\begin{array}{c}\text { hi } \\
\text { acumulada }\end{array}$ & $\begin{array}{c}\text { Acumulado } \\
\text { por nivel }\end{array}$ & $\begin{array}{c}\% \text { por } \\
\text { nivel }\end{array}$ & $\begin{array}{c}\% \\
\text { acumulado } \\
\text { por nivel }\end{array}$ \\
\hline 0 & 0 & 0 & 0 & 0 & 0 & 0 & 0 & 0 & & \\
\hline 1 & 5030 & 37195 & $\$ 21,112.33$ & 13219 & 13219 & $95.53 \%$ & $95.5 \%$ & $\$ 279,083,934$ & $87.2 \%$ & $87.2 \%$ \\
\hline 2 & 37196 & 69360 & $\$ 53,278.00$ & 481 & 13700 & $3.48 \%$ & $99.0 \%$ & $\$ 25,626,718$ & $8.0 \%$ & $95.2 \%$ \\
\hline 3 & 69361 & 101526 & $\$ 85,443.67$ & 97 & 13797 & $0.70 \%$ & $99.7 \%$ & $\$ 8,288,036$ & $2.6 \%$ & $97.8 \%$ \\
\hline 4 & 101527 & 133692 & $\$ 117,609.33$ & 21 & 13818 & $0.15 \%$ & $99.9 \%$ & $\$ 2,469,796$ & $0.8 \%$ & $98.6 \%$ \\
\hline 5 & 133693 & 165857 & $\$ 149,775.00$ & 8 & 13826 & $0.06 \%$ & $99.9 \%$ & $\$ 1,198,200$ & $0.4 \%$ & $98.9 \%$ \\
\hline 6 & 165858 & 198023 & $\$ 181,940.67$ & 1 & 13827 & $0.01 \%$ & $99.9 \%$ & $\$ 181,941$ & $0.1 \%$ & $99.0 \%$ \\
\hline 7 & 198024 & 230189 & $\$ 214,106.33$ & 4 & 13831 & $0.03 \%$ & $99.9 \%$ & $\$ 856,425$ & $0.3 \%$ & $99.3 \%$ \\
\hline 8 & 230190 & 262354 & $\$ 246,272.00$ & 3 & 13834 & $0.02 \%$ & $100.0 \%$ & $\$ 738,816$ & $0.2 \%$ & $99.5 \%$ \\
\hline 9 & 262355 & 294520 & $\$ 278,437.67$ & 1 & 13835 & $0.01 \%$ & $100.0 \%$ & $\$ 278,438$ & $0.1 \%$ & $99.6 \%$ \\
\hline 10 & 294521 & 326686 & $\$ 310,603.33$ & 0 & 13835 & $0.00 \%$ & $100.0 \%$ & $\$ 0$ & $0.0 \%$ & $99.6 \%$ \\
\hline 11 & 326687 & 358851 & $\$ 342,769.00$ & 0 & 13835 & $0.00 \%$ & $100.0 \%$ & $\$ 0$ & $0.0 \%$ & $99.6 \%$ \\
\hline 12 & 358852 & 391017 & $\$ 374,934.67$ & 0 & 13835 & $0.00 \%$ & $100.0 \%$ & $\$ 0$ & $0.0 \%$ & $99.6 \%$ \\
\hline
\end{tabular}




\begin{tabular}{|c|c|c|c|c|c|c|c|c|c|c|}
\hline $\mathbf{1 3}$ & 391018 & 423183 & $\$ 407,100.33$ & 1 & 13836 & $0.01 \%$ & $100.0 \%$ & $\$ 407,100$ & $0.1 \%$ & $99.7 \%$ \\
\hline $\mathbf{1 4}$ & 423184 & 455348 & $\$ 439,266.00$ & 1 & 13837 & $0.01 \%$ & $100.0 \%$ & $\$ 439,266$ & $0.1 \%$ & $99.9 \%$ \\
\hline $\mathbf{1 5}$ & 455349 & 487514 & $\$ 471,431.67$ & 1 & 13838 & $0.01 \%$ & $100.0 \%$ & $\$ 471,432$ & $0.1 \%$ & $100.0 \%$ \\
\hline & & & & $\mathbf{1 3 8 3 8}$ & & $100.00 \%$ & & & $100.0 \%$ & \\
\hline
\end{tabular}

Fuente: Elaboración propia con datos emitidos en la ENIGH 2018.

\section{RESULTADOS Y DISCUSIÓN}

Con apoyo en el tratamiento estadístico de los datos anteriormente expuestos, se determinó el índice de desigualdad de la percepción económica denominada aguinaldo en México, soportándose en la representación gráfica de la curva de Lorenz y el cálculo índice de Gini.

Precisamente al respecto, la curva de Lorenz ha sido utilizada tradicionalmente para representar la desigualdad, la cual fue propuesta en 1905 con el objetivo de exhibir gráficamente la falta de equilibrio en la distribución de la salud, extendiéndose postreramente en los análisis de la desigualdad económica al representar el porcentaje acumulado de ingreso (\% $\left.\mathrm{Y}_{\mathrm{i}}\right)$ recibido por un grupo de población $\left(\% \mathrm{P}_{\mathrm{i}}\right)$ y ordenado en forma ascendente en atención a la cantidad del ingreso obtenido (y1 $\leq \mathrm{y} 2 \leq \ldots, \leq \mathrm{yn})$ (Medina, 2001:14).

Dicha curva de Lorenz sin lugar a dudas es una herramienta por excelencia para ilustrar la concentración existente del ingreso, sin embargo, en diversos estudios es conveniente situar en el análisis un valor numérico que represente tal escenario con el objeto de facilitar la comparación con otras distribuciones (Reyna, 2010:57); siendo para el caso que nos ocupa el índice de Gini uno de los más utilizados en las investigaciones de la desigualdad y que se basa en la curva de Lorenz para construir dicho coeficiente, capturando el área entre la curva y una distribución plenamente equitativa. En caso de que no exista diferencia entre estas dos, dicho coeficiente es igual a 0 , lo que implica una igualdad perfecta, mientras que, al mostrarse muy separadas, dicho factor puede ser igual a 1, que representa una completa desigualdad en la distribución del ingreso (INEI, 2006:12), (Banco Mundial). La curva de Lorenz se graficó mediante la herramienta de Excel, con las opciones de gráfica de dispersión de mercado, con opción de un polinomio de orden 2, seleccionado las opciones para que se visualice la ecuación y la $r^{2}$, tal y como se observa con los datos calculados en los procesos anteriores.

Tabla 7. Concentrado de datos para gráfica de curva de Lorenz.

\begin{tabular}{|c|c|c|c|c|c|}
\hline \multicolumn{2}{|c|}{ Añ̄o 2015} & \multicolumn{2}{|c|}{ Año 2016} & \multicolumn{2}{|c|}{ Añ̄o 2018} \\
\hline hi acumulada & $\begin{array}{c}\text { \% acumulado } \\
\text { por nivel }\end{array}$ & $\begin{array}{c}\text { hi } \\
\text { acumulada }\end{array}$ & $\begin{array}{c}\% \\
\text { acumulado } \\
\text { por nivel }\end{array}$ & $\begin{array}{c}\text { hi } \\
\text { acumulada }\end{array}$ & $\begin{array}{c}\% \\
\text { acumulado } \\
\text { por nivel }\end{array}$ \\
\hline 0 & 0 & 0 & 0 & $0 \%$ & $0 \%$ \\
\hline $96.7 \%$ & $89.5 \%$ & $99.1 \%$ & $96.8 \%$ & $95.5 \%$ & $87.2 \%$ \\
\hline $99.5 \%$ & $97.5 \%$ & $99.9 \%$ & $99.3 \%$ & $99.0 \%$ & $95.2 \%$ \\
\hline $99.9 \%$ & $99.3 \%$ & $100.0 \%$ & $99.7 \%$ & $99.7 \%$ & $97.8 \%$ \\
\hline $100.0 \%$ & $99.6 \%$ & $100.0 \%$ & $99.8 \%$ & $99.9 \%$ & $98.6 \%$ \\
\hline $100.0 \%$ & $99.8 \%$ & $100.0 \%$ & $99.8 \%$ & $99.9 \%$ & $98.9 \%$ \\
\hline $100.0 \%$ & $99.9 \%$ & $100.0 \%$ & $99.8 \%$ & $99.9 \%$ & $99.0 \%$ \\
\hline $100.0 \%$ & $99.9 \%$ & $100.0 \%$ & $99.9 \%$ & $99.9 \%$ & $99.3 \%$ \\
\hline $100.0 \%$ & $99.9 \%$ & $100.0 \%$ & $99.9 \%$ & $100.0 \%$ & $99.5 \%$ \\
\hline $100.0 \%$ & $99.9 \%$ & $100.0 \%$ & $99.9 \%$ & $100.0 \%$ & $99.6 \%$ \\
\hline $100.0 \%$ & $99.9 \%$ & $100.0 \%$ & $99.9 \%$ & $100.0 \%$ & $99.6 \%$ \\
\hline $100.0 \%$ & $99.9 \%$ & $100.0 \%$ & $99.9 \%$ & $100.0 \%$ & $99.6 \%$ \\
\hline $100.0 \%$ & $99.9 \%$ & $100.0 \%$ & $99.9 \%$ & $100.0 \%$ & $99.6 \%$ \\
\hline $100.0 \%$ & $99.9 \%$ & $100.0 \%$ & $99.9 \%$ & $100.0 \%$ & $99.7 \%$ \\
\hline $100.0 \%$ & $99.9 \%$ & $100.0 \%$ & $99.9 \%$ & $100.0 \%$ & $99.9 \%$ \\
\hline $100.0 \%$ & $99.9 \%$ & $100.0 \%$ & $99.9 \%$ & $100.0 \%$ & $100.0 \%$ \\
\hline $100.0 \%$ & $99.9 \%$ & $100.0 \%$ & $99.9 \%$ & & \\
\hline $100.0 \%$ & $100.0 \%$ & $100.0 \%$ & $100.0 \%$ & & \\
\hline
\end{tabular}

Fuente: 
Elaboración propia con datos emitidos en la ENIGH 2015, 2016 y 2018.

Una vez obtenida la curva de Lorenz, se puedo estimar que la distribución del aguinaldo en los tres años no es igualitaria, ya que, para una distribución totalmente igual, debería representarse una recta de 45 grados, donde $\mathrm{x}=\mathrm{y}$, como se muestra en las siguientes gráficas.

Gráfica 1. Distribución del aguinaldo 2015 en México.

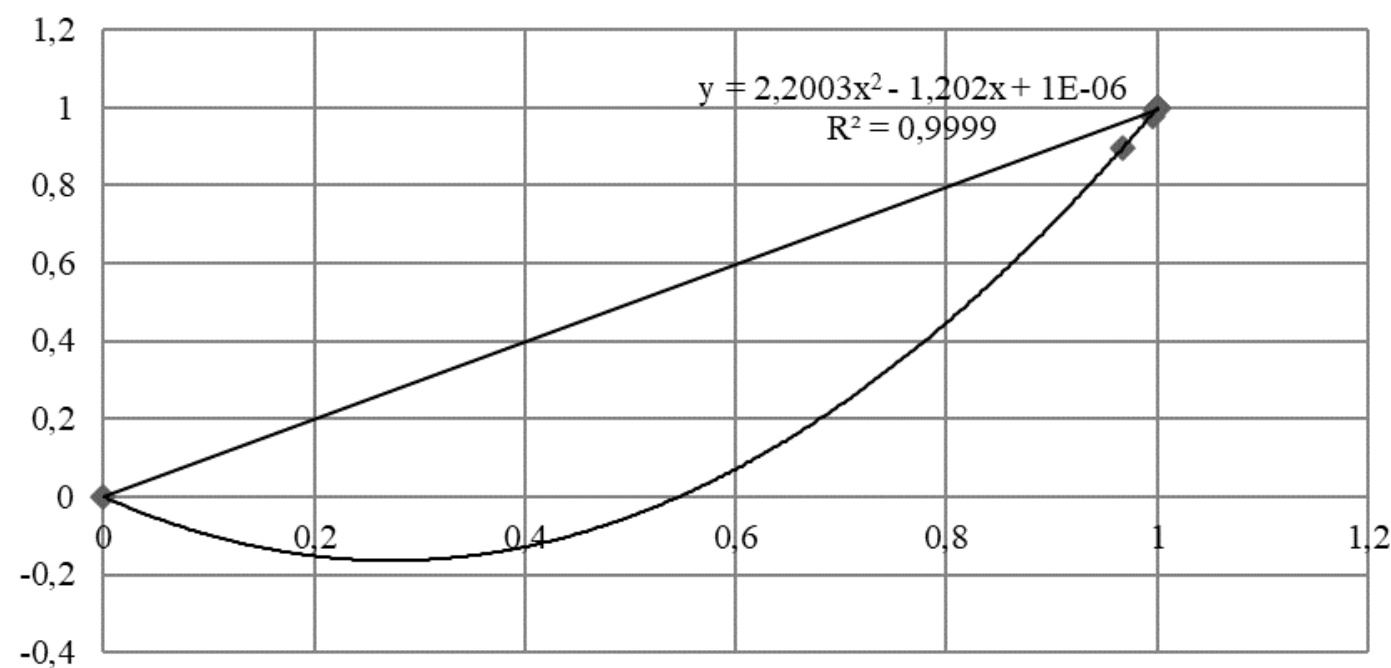

- \% acumulado por nivel

— Polinomial (\% acumulado por nivel)

—Linear (\% acumulado por nivel)

Fuente: Elaboración propia con datos emitidos en la ENIGH 2015.

Gráfica 2. Distribución del aguinaldo 2016 en México.

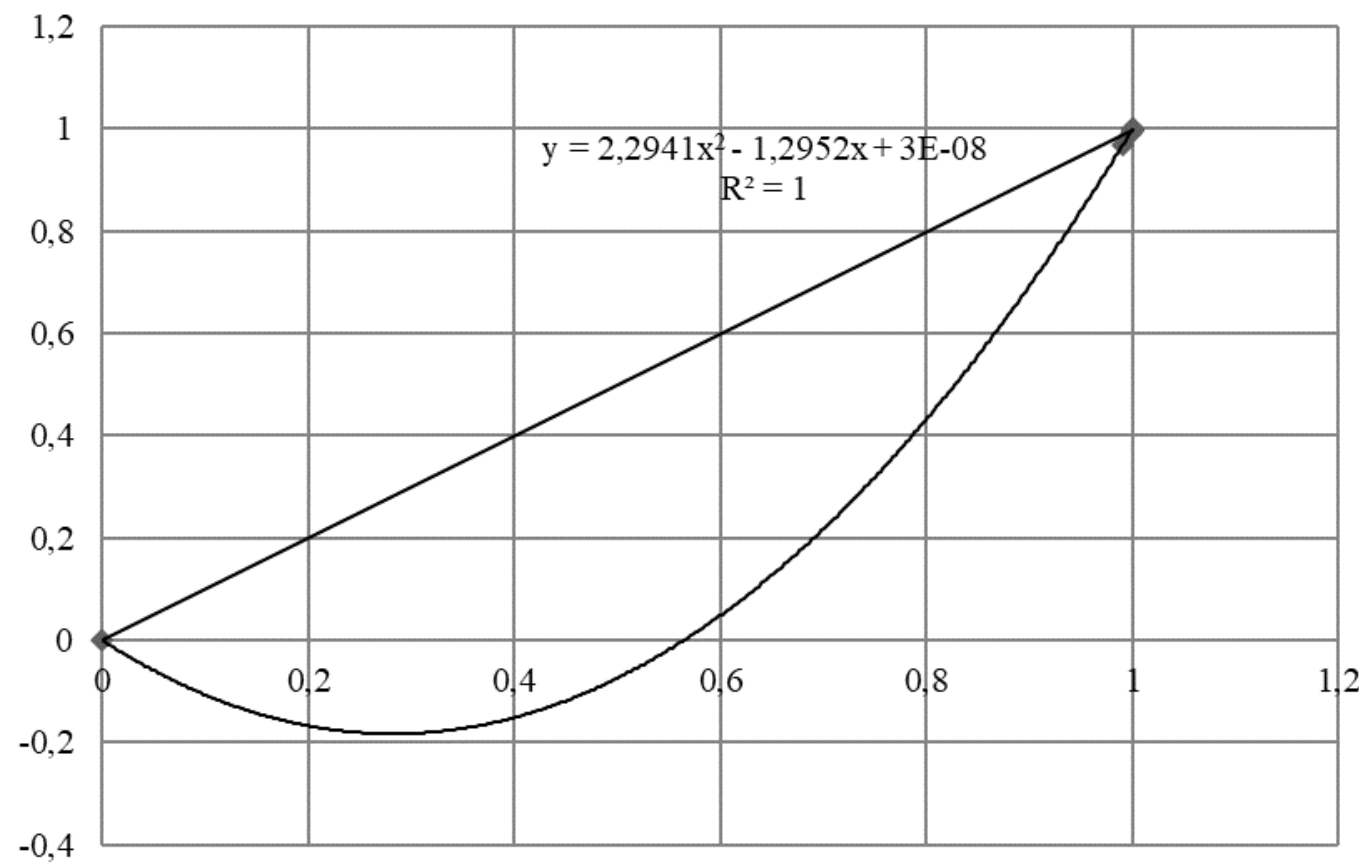

- \% acumulado por nivel

- Polinomial (\% acumulado por nivel)

— Linear (\% acumulado por nivel)

Fuente: Elaboración propia con datos emitidos en la ENIGH 2016. 
Gráfica 3. Distribución del aguinaldo 2018 en México.

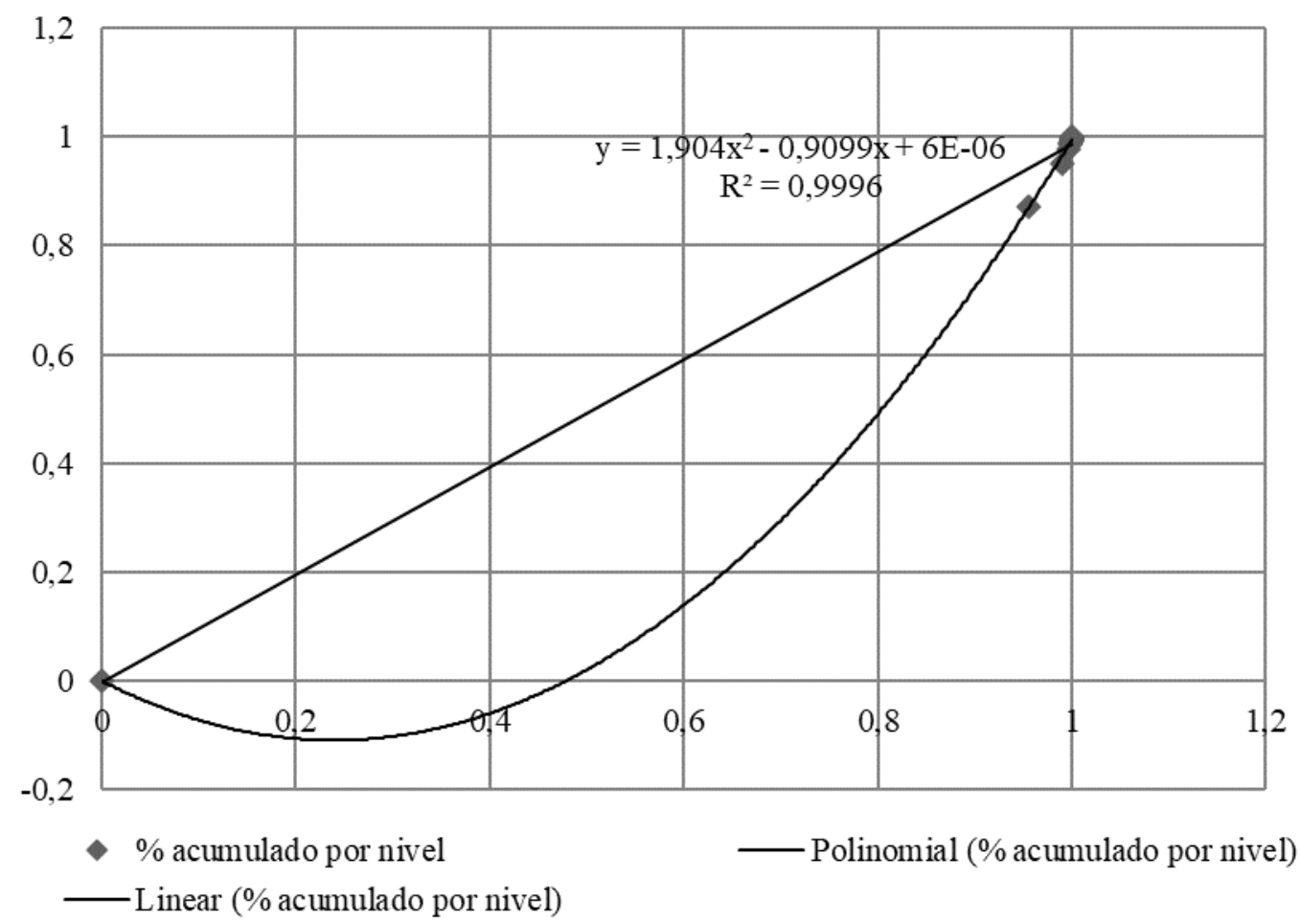

Fuente: Elaboración propia con datos emitidos en la ENIGH 2018.

Para conocer que tan desigual fue la distribución del aguinaldo por los períodos analizados numéricamente, se determinó el índice de Gini, es decir, el área que existe entre la línea recta y la curva de Lorenz. De tal manera que, si hubiera una desigualdad perfecta el área que se observara fuera similar a un triángulo. A mayor área, mayor desigualdad.

De tal forma que, para deducir el área existente mediante la integración de las ecuaciones expresadas en los gráficos anteriores, se soportó en el solucionador matemático Symbolab, multiplicándose el resultado obtenido por 2 y obteniéndose el índice de Gini por cada uno de los períodos analizados.

Tabla 8. Resumen curva de Lorenz e índice de Gini 2015, 2016 y 2018.

\begin{tabular}{|c|c|c|c|c|}
\hline Año & $\begin{array}{c}\text { Línea recta que } \\
\text { representa igualdad } \\
\text { perfecta }\end{array}$ & Curva de Lorenz & Área & $\begin{array}{c}\text { Multiplicado por 2 } \\
\text { = Índice de Gini }\end{array}$ \\
\hline 2015 & $\mathrm{X}=\mathrm{Y}$ & $\mathrm{Y}=2.2003 \mathrm{x}^{2}-1.202 \mathrm{x}+1 \mathrm{E}-06$ & 0.36756 & 0.73512 \\
\hline 2016 & $\mathrm{X}=\mathrm{Y}$ & $\mathrm{Y}=2.2941 \mathrm{x}^{2}-1.2952 \mathrm{x}+3 \mathrm{E}-08$ & 0.38290 & 0.76580 \\
\hline 2018 & $\mathrm{X}=\mathrm{Y}$ & $\mathrm{y}=1.904 \mathrm{x}^{2}-0.9099 \mathrm{x}+6 \mathrm{E}-06$ & 0.32028 & 0.64056 \\
\hline
\end{tabular}

Fuente: Elaboración propia con datos emitidos en la ENIGH 2015, 2016 y 2018. 
En el caso del aguinaldo en México es un ingreso muy significativo en los hogares, pues dicha percepción económica se espera obtener con gran entusiasmo y expectativa en el mes de diciembre de cada año con el objetivo de cubrir deudas o solventar las necesidades de la familia de los individuos que perciben tal remuneración, la cual estará supeditada al salario diario del trabajador y de los días que el patrón esté dispuesto a liquidar al mismo con sustento en el contrato colectivo o individual según sea el caso; no debiendo ser inferior a los 15 días que se estipulan en la LFT.

Por tal motivo, con la existencia de la amplia brecha salarial, la desigualdad en los distintos grupos de trabajo, las características contractuales y entre otros factores que incurren, como se puede valorar en los datos anteriormente expuestos, tanto en los gráficos de la curva de Lorenz como en el coeficiente de Gini en la dispersión económica del aguinaldo por los años 2015, 2016 y 2018 en México, refleja una alta desigualdad en dicha percepción en los hogares y que se estima su pago en función del salario diario vigente al momento de su determinación del trabajador; mostrándose además que el año 2016 fue más inequitativo con una leve disminución en el año 2018.

Asimismo, es preciso no soslayar todos aquellos factores que inciden en la desigualdad salarial, pues sería muy limitativo contemplar solamente los aspectos contractuales y el salario del trabajador, en virtud de que existen distintos análisis empíricos que exponen tales elementos que influyen directamente a tal remuneración y por ende en las distintas percepciones económicas como en el caso del aguinaldo. Verbigracia, Varela y Urciaga (2012) concluyen que la inversión en capital humano mediante la educación formal es un elemento esencial en los niveles de ingresos de los jefes de los hogares en México, toda vez que fortalece las condiciones de bienestar social al obtener mejores remuneraciones. De igual forma, Carrera, Villafuerte y Rodríguez (2017) comparten en su estudio que la endeble infraestructura del Estado para coadyuvar al esquema del desarrollo humano y en muchos de los casos el bajo grado de inversión en capacitación de los trabajadores por parte de los empleadores, agudizan la desigualdad en el ingreso.

\section{CONCLUSIÓN}

Como se puede apreciar, el estudio se limitó a una de las percepciones económicas de los hogares en México, quedando evidente la fundamentación jurídica del derecho laboral al aguinaldo, remuneración económica que está sujeta al salario diario del trabajador y a los días que esté dispuesto el patrón a pagar conforme a la relación contractual individual o colectiva según sea el caso, no debiendo ser inferior a 15 días de la cuota diaria. De igual forma y sin duda alguna, con los datos antes expuestos mediante la presentación gráfica de curva de Lorenz y el índice Gini, exhiben una alta desigualdad en dicha distribución pecuniaria en el país en los periodos analizados, de tal manera que la baja percepción en la remuneración del aguinaldo va acompañada a los bajos salarios así como a la desigualdad de los grupos de los trabajadores, en virtud de que existen empleados que además de percibir un mejor salario obtienen una mayor cantidad de días del ingreso en estudio, reflejándose con ello una amplia brecha en la desigualdad de los hogares en México.

Tal análisis refleja la congruencia con los diversos estudios empíricos que se han expuesto en correlación a la brecha o desigualdad salarial en México desde sus diversos enfoques, los cuales se apoyan metodológicamente en su mayoría en el índice de Gini, coincidiendo en gran medida con dichos análisis en que el fenómeno de la desigualdad es muy complejo y difícil de erradicar, escenario donde la concentración de la riqueza, la distribución del ingreso, la pobreza, la carencia en la educación y su incidencia en el capital humano están estrechamente relacionados; sin embargo, es imperante la implementación de políticas públicas orientadas a disminuirla y por ende combatir sus efectos nocivos en el desarrollo de las comunidades. 
Por lo tanto, sirva el presente análisis para posteriores reflexiones experimentales en correlación a la indagación de las desigualdades en las distintas percepciones económicas y de manera específica que obtienen los distintos grupos de individuos en los hogares en México; tales como, vacaciones, bonos de puntualidad y asistencia, prima dominical, horas extras, bonos de despensa, entre otros ingresos vinculantes a la relación laboral individual o colectiva, así como aquellas que se obtienen una vez que se rescinde el mismo vínculo contractual, tales como las indemnizaciones, las pensiones y con posible perspectiva de género.

\section{REFERENCIAS}

Araújo, A. (2015). La desigualdad salarial de género medida por regresión cuantílica: el impacto del capital humano, cultural y social. Revista Mexicana de Ciencias Políticas y Sociales, vol. LX, núm. 223, enero-abril, 2015, pp. 287315. [En línea]. Disponible en: https://www.redalyc.org/articulo.oa?id=42132 948011 [Extraído el 18 de febrero 2021].

Banco Mundial. LAC Equity Lab: Desigualdad Distribución de Ingresos. [En línea]. Disponible en: https://www.bancomundial.org/es/topic/povert y/lac-equity-lab1/income-inequality/incomedistribution [Consultado el 9 de febrero 2021].

Carrera, O., Villafuerte, L. y Rodríguez A. (2017). Desigualdad en México bajo el enfoque de una Economía de Mercado Jerárquica (EMJ). Ensayos de Economía, No. 51 julio-diciembre. [En línea]. Disponible en: http://dx.doi.org/10.15446/ede.v27n51.69108 [Extraído el 27 enero 2021].

Castro, D. y Huesca, L. (2007). Desigualdad salarial en México: una revisión. Papeles de Población No. 54, pp. 225-264. [En línea]. Disponible en:

http://www.scielo.org.mx/pdf/pp/v13n54/v13n 54a9.pdf [Extraído el 10 de febrero 2021].

CNDH. Salario mínimo y derechos humanos. [En línea]. Disponible en: https://www.cndh.org.mx/sites/all/doc/Inform es/Especiales/Salario-Minimo-DH.pdf [Extraído el 15 de febrero 2021].

CPEUM. Constitución publicada en el Diario Oficial de la Federación el 5 de febrero de 1917. Última reforma publicada en el Diario Oficial de la Federación el 19 de febrero del 2021. [En línea]. Disponible en: http://www.diputados.gob.mx/LeyesBiblio/ref /cpeum.htm [Extraída el 20 de febrero del 2021].

Hernández, L. (2020). México es el país de la OCDE con mayor desigualdad económica. Economía, el Financiero. [En línea]. Disponible en: https://www.elfinanciero.com.mx/economia/m exico-es-el-pais-de-la-ocde-con-mayordesigualdad-economica [Consultado el 20 de febrero 2021].

INEGI. Encuesta Nacional de Ingresos y Gastos en los Hogares (ENIGH). [En línea]. Disponible en: https://www.inegi.org.mx/datos/?ps=Programa s [Extraído el 28 de enero 2021].

INEI (2006). Glosario básico de términos estadísticos. Centro de Investigación y Desarrollo. [En línea]. Disponible en: http://chamilo.cut.edu.mx:8080/chamilo/cours es/ESTADISTICA2019/document/glosario_de _estadistica.pdf [Extraído el 10 de febrero 2021].

Jasso, I. y Acevedo, G. (2004). La brecha salarial en México con enfoque de género: capital humano, discriminación y selección muestral. Ciencia UANL, vol. 7 No. 1 enero-marzo, pp. 66-71. [En línea]. Disponible en: http://eprints.uanl.mx/1521/1/brecha_salarial.p df [Extraído el 18 de febrero 2021].

LFT. Nueva Ley publicada en el Diario Oficial de la Federación el $1^{\circ}$ de abril de 1970. Última reforma publicada en el Diario Oficial de la Federación el 11 de enero de 2021. [En línea]. Disponible en: http://www.diputados.gob.mx/LeyesBiblio/ref /lft.htm [Extraída el 20 de febrero del 2021].

Llamas, L., Huesca, L. y Gutiérrez, L. (2019). Desigualdad y justicia en la remuneración por género en México y la frontera norte. Análisis Económico, vol. XXXIV, núm. 86, mayoagosto, pp. 91-112. [En línea]. Disponible en: http://www.analisiseconomico.azc.uam.mx/in dex.php/rae/article/view/418/329 [Extraído el 16 de febrero 2021].

Medina, F. (2001). Consideraciones sobre el índice de Gini para medir la concentración del ingreso. CEPAL - SERIE No. 9 Estudios estadísticos y prospectivos. Publicación de las Naciones 
Unidas. [En línea]. Disponible en: https://repositorio.cepal.org/bitstream/handle/1 1362/4788/1/S01020119_es.pdf [Extraído el 11 de febrero 2021].

Navarrete, J. (2016). ¿Desigualdad y crecimiento? Economíaunam, vol. 13, núm. 37, enero-abril, pp. 45-73 [En línea]. Disponible en: http://www.revistas.unam.mx/index.php/ecu/a rticle/view/54737/48646 [Extraído el 25 de enero 2021].

ONU. Declaración Universal de los Derechos Humanos. [En línea]. Disponible en: https://www.un.org/es/documents/udhr/UDHR _booklet_SP_web.pdf [Extraída el 15 de febrero del 2021].

REA. Aguinaldo. [En línea]. Disponible en: https://dle.rae.es/aguinaldo [Consultada el 21 de febrero del 2021].

Reyna, A. (2010). Estadística descriptiva para ciencias sociales. Instituto de Ciencias Sociales y Humanidades. Universidad Autónoma del Estado de Hidalgo.

Rodríguez, R. (2018). Brecha salarial por género en México: Desde un enfoque regional, según su exposición a la apertura comercial 2005-2015. Nóesis. Revista de Ciencias Sociales y Humanidades, vol. 27, núm. 54. [En línea]. Disponible en: http://erevistas.uacj.mx/ojs/index.php/noesis/a rticle/view/1147/pdf_42 [Extraído el 16 de febrero 2021].

Rodríguez, R. y Limas, M. (2017). El análisis de las diferencias salariales y discriminación por género por áreas profesionales en México, abordado desde un enfoque regional, 2015. Estudios Sociales, vol. 27, núm. 49, enerojunio, pp. 121-150. [En línea]. Disponible en: http://www.scielo.org.mx/pdf/estsoc/v27n49/0 188-4557-estsoc-27-49-00121.pdf [Extraído el 16 de febrero 2021].

Sánchez, R. (2009). Origen histórico-jurídico del aguinaldo: del strenna romano al salario en especie. En Francisco Javier Campos y Fernández de Sevilla (Coord.). La Natividad, arte, religiosidad y tradiciones populares; pp. 715-730. [En línea]. Disponible en: https://dialnet.unirioja.es/servlet/articulo?codi go=3041014 [Extraído el 20 de febrero 2021].

Sturges, H. (1926). The choice of a class-interval. J. Amer. Statist. Assoc. 21: 65-66.

Symbolab. [En línea]. Disponible en: https://es.symbolab.com/ [Consultado el $16 \mathrm{de}$ febrero 2021].
Varela, R. y Urciaga, J. (2012). Diferencias salariales en México: una perspectiva de educación y actividad económica. Revista de la Educación Superior, vol. XLI (2), núm. 162, abril-junio, pp. 25-43. [En línea]. Disponible en: https://www.redalyc.org/articulo.oa? $i d=60425$ 033002 [Extraído el 17 de febrero 2021]. 